\title{
Dual-scope of circadian rhythm biology
}

\section{Kazuhiro Yagita ${ }^{1}$}

Published online: 6 December 2017

(c) Japanese Society of Sleep Research 2017

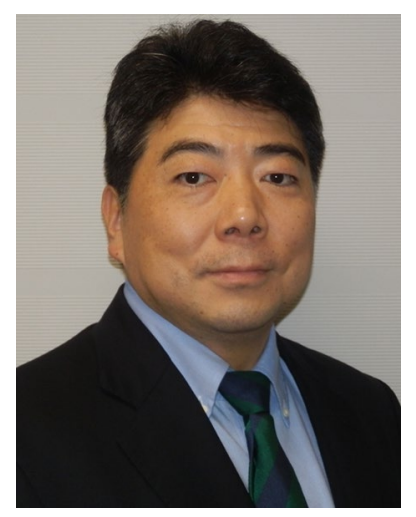

Three scientists, Jeffrey C. Hall, Michael Rosbash and Michael W. Young, were awarded the Nobel Prize in Physiology and Medicine 2017 for their discoveries of molecular mechanisms controlling the circadian rhythm. They are famous circadian biologists and are known as the "legends" who cloned Drosophila clock genes and established the concept of the "transcriptional/translational feedback loop (TTFL) model." In various organisms, including mammals, TTFL composed of clock genes was found, and now it has become a central dogma of the molecular mechanism of the circadian clock.

Circadian clocks regulate the daily fluctuations of essential biological processes on a molecular and organismal level to predict and adapt to the cyclic environment of the rotating earth. In mammals, cell-autonomous circadian clocks exist in both the suprachiasmatic nucleus (SCN) and peripheral cells throughout the body, suggesting that circadian clocks function as an interface connecting cyclic environmental changes and physiology from a cellular to organismal level. Since the discovery of the first clock

Kazuhiro Yagita

kyagita@koto.kpu-m.ac.jp

1 Department of Physiology and Systems Bioscience, Kyoto Prefectural University of Medicine, Kawaramachi-Hirokoji, Kamigyo-ku, Kyoto 602-8566, Japan gene, period, in Drosophila, we have learned that clock genes regulate the molecular clock and circadian rhythm.

Winning the Nobel Prize for circadian biology was very good news and made us very happy. However, we also realize that un-solved important questions still remain. One is the basic point of view, while another is circadian rhythm science for social and public health. Although molecular mechanisms of circadian clock have recently been elucidated, we cannot fully answer how circadian clocks generate $\sim 24 \mathrm{~h}$, nor do we know how the circadian clocks are entrained by the environmental cycle. These questions must be solved not only to understand the principle of circadian clock, but also to provide knowledge preventing various health disorders or chronic diseases. Evidence has been accumulating showing that the risks of various diseases such as metabolic syndrome, cardiovascular events and some types of cancers are increased in shift workers with circadian disorder [1-3]. In Japan, it has been reported that the number of shift workers, including night workers, are greater than 10 million [4]. Although there is no doubt about the correlation between circadian rhythm disorder and various chronic diseases, effective measures or solutions available for the social and public health have not yet been established.

The time has come. Circadian rhythm biology, as well as sleep biology, is encouraged to have a scope looking at the "real world". Accumulated information from the basic sciences should be translated into social and public health benefits. In addition, epidemiological or clinical evidence of correlation may provide important scientific seeds contributing to understanding the principles of circadian rhythms. Dual-scope science with basic and social points of view may accelerate the research into sleep and biological rhythms.

\section{References}

1. Knutsson A. Health disorders of shift workers. Occup Med (Lond). 2003;53:103-8. 
2. Wang XS, Armstrong ME, Cairns BJ, Key TJ, Travis RC. Shift work and chronic disease: the epidemiological evidence. Occup Med (Lond). 2011;61:78-89.

3. Golombek DA, Casiraghi LP, Agostino PV, Paladino N, Duhart JM, Plano SA, et al. The times they're a-changing: effects of circadian desynchronization on physiology and disease. J Physiol Paris. 2013;107:310-22.

4. Kubo T. Estimate of the number of night shift workers ins Japan. J UOEH. 2014;36:273-6. 